\title{
Implications of Redesigned, High-Radiative-Efficiency GaInP Junctions on III-V Multijunction Concentrator Solar Cells
}

\author{
John F. Geisz, Myles A. Steiner, Iván García, Ryan M. France, Daniel J. Friedman, and Sarah R. Kurtz
}

\begin{abstract}
Nonradiative recombination in inverted GaInP junctions is dramatically reduced using a rear-heterojunction design rather than the more traditional thin-emitter homojunction design. When this GaInP junction design is included in inverted multijunction solar cells, the high radiative efficiency translates into both higher subcell voltage and high luminescence coupling to underlying subcells, both of which contribute to improved performance. Subcell voltages within two and four junction devices are measured by electroluminescence and the internal radiative efficiency is quantified as a function of recombination current using optical modeling. The performance of these concentrator multijunction devices is compared with the Shockley-Queisser detailed-balance radiative limit, as well as an internal radiative limit, which considers the effects of the actual optical environment in which a perfect junction may exist.
\end{abstract}

Index Terms-Luminescent coupling, multijunction photovoltaic cells, radiative efficiency, III-V.

\section{INTRODUCTION}

$\mathbf{S}$ HOCKLEY and Queisser (SQ) described fundamental limits for the performance of solar cells based on detailed balance arguments [1]. Rau [2] and others [3], [4] have shown that any reduction from this "detailed balance" limit can be quantified simply by the external radiative efficiency (ERE or $\eta_{\text {ext }}$ ). Thus, a reciprocal relationship between the voltage across a solar cell junction (which excludes series resistance) and the efficiency at which it emits light can be obtained at any recombination current density, $J$

$$
V(J)=\frac{k T}{q} \ln \left[\frac{J \times \eta_{\text {ext }}}{J_{0}^{\text {rad }}}+1\right]
$$

where $k$ is the Boltzmann constant, $T$ is the solar cell temperature, $q$ is the elemental charge, and the SQ dark current density of a particular junction in the external radiative limit can be calculated from the external quantum efficiency (EQE)

$$
J_{0}^{\mathrm{rad}}=\int_{0}^{\infty} q \phi_{\text {black body }}(T, E) \mathrm{EQE}(E) d E
$$

where $\phi_{\text {black body }}$ is Planck's black body emission spectrum and $E$ is the photon energy. (Note that the +1 term in (1) is often neglected.) The task of maximizing the solar cell voltage, therefore, amounts to maximizing ERE. In addition, these expressions allow the determination of junction voltages through electroluminescence (EL) measurements [5]-[7].

Light emitted by radiative recombination in a solar cell junction can be reabsorbed within the junction, absorbed elsewhere in the structure, reflected back to the junction (often by total internal reflection), or completely escape out the front. When the emitted light is absorbed within another junction, the effect known as luminescent coupling (LC) [8], is observed. Optical modeling of the actual solar cell layer structure can be used to relate the ERE to an internal radiative efficiency (IRE or $\eta_{\text {int }}$ ) assuming uniform emission and given accurate optical constants $n(\lambda), k(\lambda)$ of each layer [9]

$$
\eta_{\text {ext }}=\frac{\eta_{\text {int }} \bar{P}_{e s c}}{1-\eta_{\text {int }} \bar{P}_{a b s}}
$$

where $\bar{P}_{e s c}$ and $\bar{P}_{a b s}$ are the average probabilities that an internally emitted photon either escapes out the front of the device or is reabsorbed within the junction, respectively.

The IRE is just the average probability that any recombination event within the junction will be radiative and is often thought of as a measure of material quality, but the IRE can be a strong function of $J$ and is affected by junction electrical structure parameters such as thickness, doping, and bandgap. It is actually an absolute measure of the junction quality that can be used to compare junctions of various materials with different band gaps and within different optical environments as a function of $\mathrm{J}$.

We have recently demonstrated significantly improved IRE ( $\sim 80 \%$ at one-sun) in inverted single-junction $1.8-\mathrm{eV}$ GaInP solar cells using a rear-heterojunction (RHJ) electrical design [7] that resulted in a record $20.8 \%$ efficiency. While the ERE, and thus voltage, of this inverted single-junction result benefited from an excellent gold back surface reflector (BSR), the high IRE of this electrical design also has significant implications when it is incorporated into multijunction concentrator solar cells. In this paper, we characterize, quantify, and discuss these implications for two- and four-junction inverted metamorphic (IMM) solar cells using this RHJ design in the top GaInP junction. We also propose and compare results with a more 

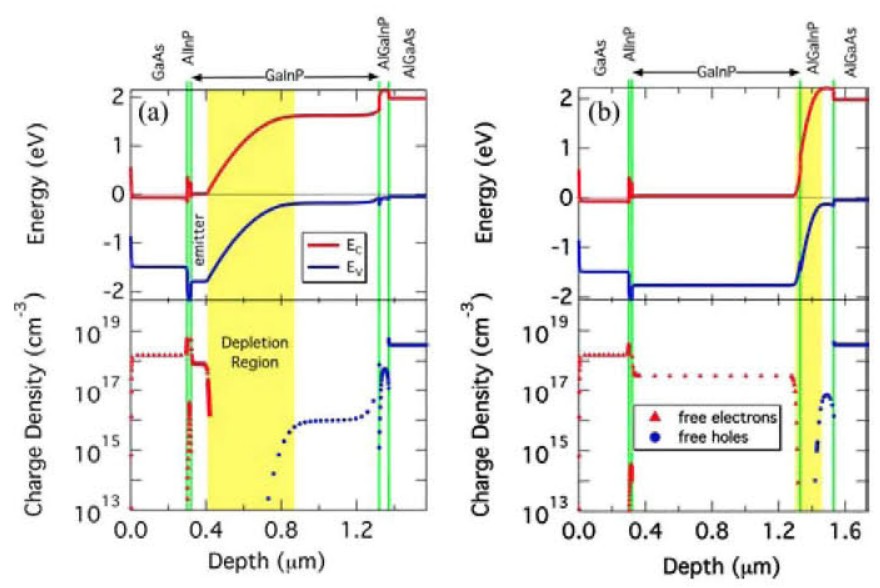

Fig. 1. Calculated open-circuit band bending and free carrier density profile for (a) traditional versus (b) RHJ designs for the GaInP junction. The depletion region is highlighted in yellow.

practically achievable radiative limit than the SQ limit (which corresponds to $100 \%$ ERE). The "internal radiative limit" corresponding to $100 \% \mathrm{IRE}$ is useful to compare the actual junction voltages with the highest achievable voltages within a given optical environment.

\section{REDESIGNING GaInP JunCtions}

The traditional electrical structure for GaInP junctions and, indeed, most modern III-V junctions has evolved over the years toward a relatively standard n-on-p design consisting of a thin, highly n-doped emitter and a thicker, more lightly p-doped base with higher band gap passivating layers at the front and back. The optimization of this traditional design was primarily driven by the need to ensure high carrier collection efficiency based on bulk material and interface transport parameters [10]. We have demonstrated a very different RHJ design for GaInP solar cells [7], which consists of a thick n-type emitter ( $[\mathrm{Si}] \sim 5 \times 10^{17} \mathrm{~cm}^{-3}$ ) and a higher band gap p-type $\mathrm{Al}_{.25} \mathrm{Ga}_{25} \operatorname{In}_{.5} \mathrm{P}$ base $\left([\mathrm{Zn}] \sim 10^{17}-10^{18} \mathrm{~cm}^{-3}\right)$. The motivation for this RJH design is focused on increasing the IRE by reducing nonradiative recombination. The calculated open-circuit band bending diagrams [11] of these two junction designs are shown in Fig. 1. Because the primary nonradiative recombination mechanism in GaInP junctions is Shockley-Read-Hall recombination via deep-level states within the depletion region as described by Sah et al. [12], we believe that moving the depletion region into the higher band gap AlGaInP base is instrumental in increasing the IRE [13]. With very little carrier generation within the $\mathrm{Al}_{25} \mathrm{Ga}_{25} \mathrm{In}_{.5} \mathrm{P}$ base, a back surface field is not needed. The primary challenge with the RHJ design is that the minority carrier diffusion length of the emitter must be greater than its thickness to prevent current loss. An additional advantage of the RHJ is that the thicker emitter results in a significantly lower sheet resistance $(100 \Omega /$ square versus $400 \Omega /$ square).

We have grown by organometallic vapor phase epitaxy (OMVPE) single-junction GaInP solar cells with $1-\mu \mathrm{m}$-thick
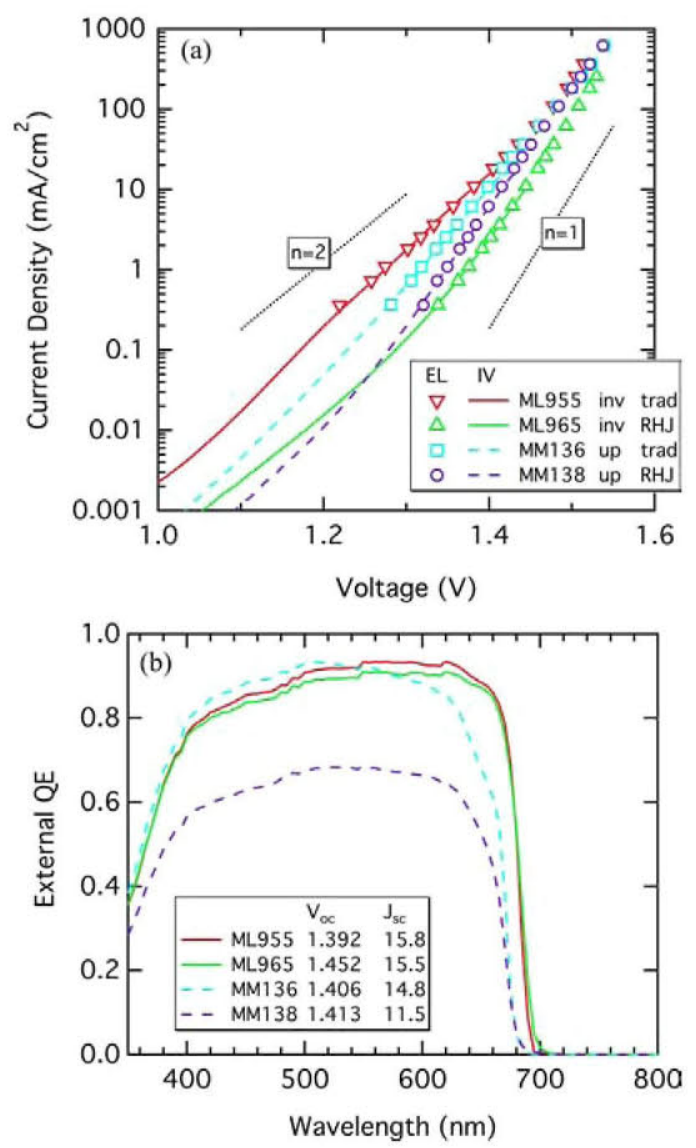

Fig. 2. Comparison of traditional versus RHJ designs, and inverted versus upright single-junction GaInP performance. (a) Dark JV from electrical and EL measurements. (b) External QE. Inset lists summary of light JV measurements.

absorber layers in both upright and inverted [14] configurations using the traditional and RHJ electrical designs. Devices were characterized by EQE, dark and light current-voltage $(I-V)$ measurements, and EL. Fig. 2 shows a comparison of results for these four GaInP solar cells. While the dark current of the RHJ upright device is lower than the traditional upright device, the $\mathrm{EQE}$ and short-circuit current density $\left(J_{\mathrm{sc}}\right)$ of the RHJ upright device are considerably degraded from the traditional upright device probably because the diffusion length in the upright material is less than the emitter thickness, justifying the traditional design for upright structures. In contrast, the RHJ inverted device enjoys a considerable reduction in dark current compared with the traditional inverted device with only a slight degradation of $\mathrm{EQE}$ or $J_{\mathrm{sc}}$. Indeed, the EQE of both inverted devices are well modeled by the absorption [15] within these structures indicating nearly perfect carrier collection efficiency. Thus, the minority carrier diffusion length of the inverted RHJ emitter must be $>1 \mu \mathrm{m}$. These results imply a significant difference in diffusion length of the RHJ emitter and/or interface quality between the inverted and upright growth. These differences likely arise from the asymmetry of atomic diffusion (particularly $\mathrm{Zn}$ ) or interface switching during the OMVPE growth. While it is also possible that the diffusion length of the inverted device is improved through photon recycling enhanced transport 


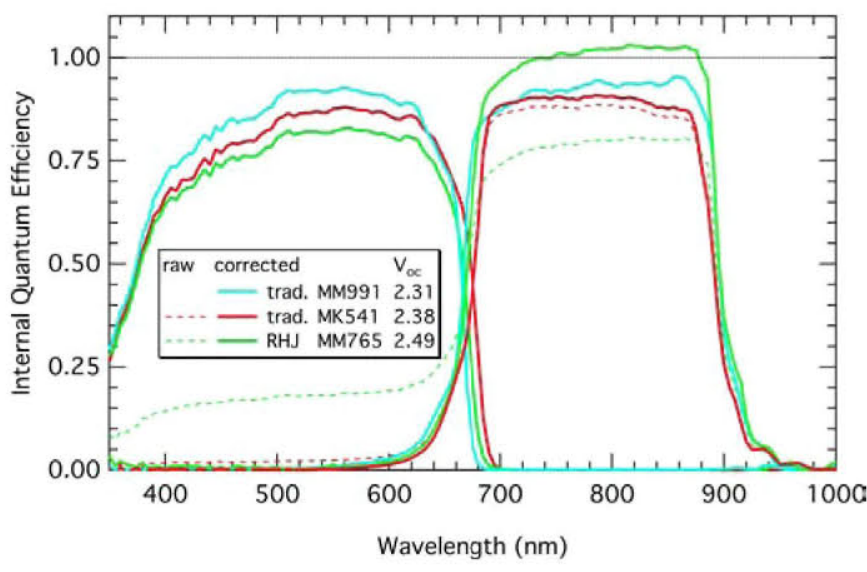

Fig. 3. IQE of inverted 2J GaInP/GaAs solar cells. Solid lines are corrected for $L C$, dashed are uncorrected.

properties [16], [17] in this single-junction device with a BSR, subsequent multijunction results indicate that this cannot be the only reason.

\section{Inverted GaInP/GAAs TANDEM SOLAR CELLs}

The simplest multijunction device to demonstrate the implications of using the RHJ compared with traditional design top GaInP junctions is an inverted two-junction (2J) GaInP/GaAs tandem. We have fabricated several inverted $2 \mathrm{~J}$ devices, again with $1-\mu \mathrm{m}$-thick 1.8 -eV GaInP absorber layers in the top junction. These inverted $2 \mathrm{~J}$ devices have a gold BSR behind the second (GaAs) junction, but not behind the top (GaInP) junction. A relatively thin $(2.0-2.5 \mu \mathrm{m}) \mathrm{GaAs}$ absorber layer is used because the BSR effectively doubles the absorption path length for this junction. Because different antireflection coatings were used, the internal quantum efficiency (IQE) rather than the EQE is compared in Fig. 3.

We have quantified the LC [8], [16], [18], [19] between junctions as well as the EL [5]-[7] in these inverted 2J devices. When significant LC occurs, the EL analysis must be corrected to account for this transfer of current between junctions [20]. The quantum efficiency must also be corrected for LC effects [18].

We compare a 2J device with a RHJ top cell (MM765, green) with two $2 \mathrm{~J}$ devices using a traditional top cell design. While MK541 (red) represents an excellent $2 \mathrm{~J}$ device using the traditional design, MM991 (blue) represents a very poorly emitting top junction using the traditional design due to overdoping. Fig. 3 shows good IQE for both junctions of all devices, indicating no gross problems with carrier transport properties. (The large LC correction for the second junction of MM765 resulted in an unphysical IQE $>1$. This artifact may indicate that other factors in addition to LC, such as shunting, may have affected the IQE, but we believe the shape of the IQE to be improved by the correction.) Fig. 4 shows measurements of the seriesconnected $J_{\mathrm{sc}}$ of the tandems while varying the illumination of the top junction with almost no external illumination onto the bottom GaAs junction. These measurements are used to quantify

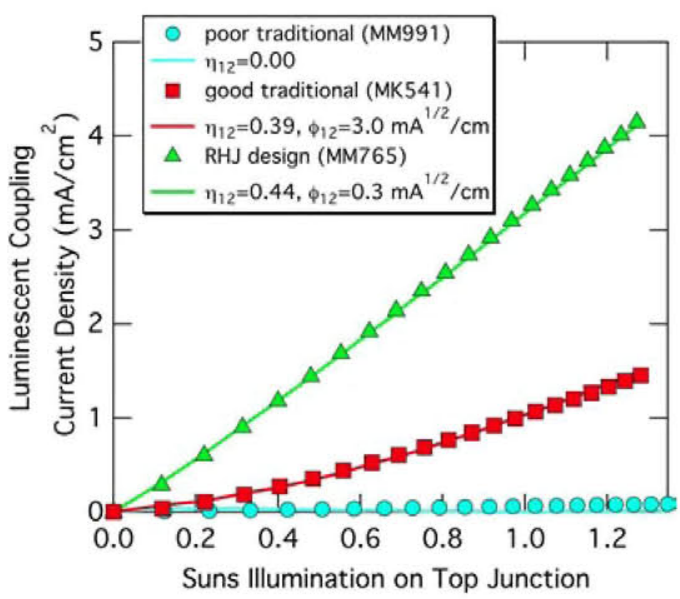

Fig. 4. LC measurements of inverted $2 \mathrm{~J} \mathrm{GaInP} / \mathrm{GaAs}$ solar cells.

the LC parameters $\eta_{12}$ and $\varphi \equiv J_{02} /\left(2 \sqrt{J_{01}}\right)$ as defined in [8] and summarized in the figure legend. While the poor traditional device (MM991) has almost no LC, the good traditional device (MK541) has fairly strong, but nonlinear LC. This nonlinear LC is characteristic of a transition between $(n=2)$ nonradiative dominated to $(n=1)$ radiative dominated recombination [8] in the top junction as the recombination current is increased which results in a large $\phi$ parameter. The RHJ tandem (MM765) has even stronger, nearly linear LC characterized by a small $\phi$ parameter. Indeed, almost half of the excess photocurrent generated in the top RHJ is transferred through LC to the GaAs junction.

Fig. 5 shows three equivalent representations [using (1)-(3)] of the LC-corrected EL data that separately characterize the top and bottom junctions as 1) $\mathrm{V}(\mathrm{J}), 2) \eta_{\text {ext }}(\mathrm{J})$, and 3) $\eta_{\text {int }}(\mathrm{J})$. The axes in Fig. 5(a) are switched to show the data as familiar dark JV curves of each junction. Along with the measured data (shown as markers), two radiative limits are shown as lines: the SQ (or $100 \%$ ERE) limits are shown as solid lines or pink highlights; and the "internal radiative limits" (or 100\% IRE) are shown as dashed lines or yellow highlights. The internal radiative limit describes the best junction performance that can be expected given the actual optical geometry used and is characterized by the optical parameters $\bar{P}_{e s c}$ and $\bar{P}_{a b s}$ used in (3) for each junction (typical values shown in Table I). These optical parameters are calculated from detailed optical modeling of the layered geometry as described in [9] using the transfer matrix method [15] and optical properties $\mathrm{n}(\lambda), \mathrm{k}(\lambda)$ of each layer. This calculation of the optical parameters assumes uniform emission within the junction, but localized emission within the junction would result in different results.

The performance of the three different top junctions within the inverted $2 \mathrm{~J}$ devices stand in stark contrast to each other. In Fig. 5(c), the IRE of the RHJ top junction (green) varies slowly from $75 \%$ to $85 \%$ over the range of current densities of about 5 to $1000 \mathrm{~mA} / \mathrm{cm}^{2}$, although it appears to begin falling rapidly below $\sim 1 \mathrm{~mA} / \mathrm{cm}^{2}$. The IRE of the poor traditional top cell (blue) is almost zero and the good traditional top junction (red) 

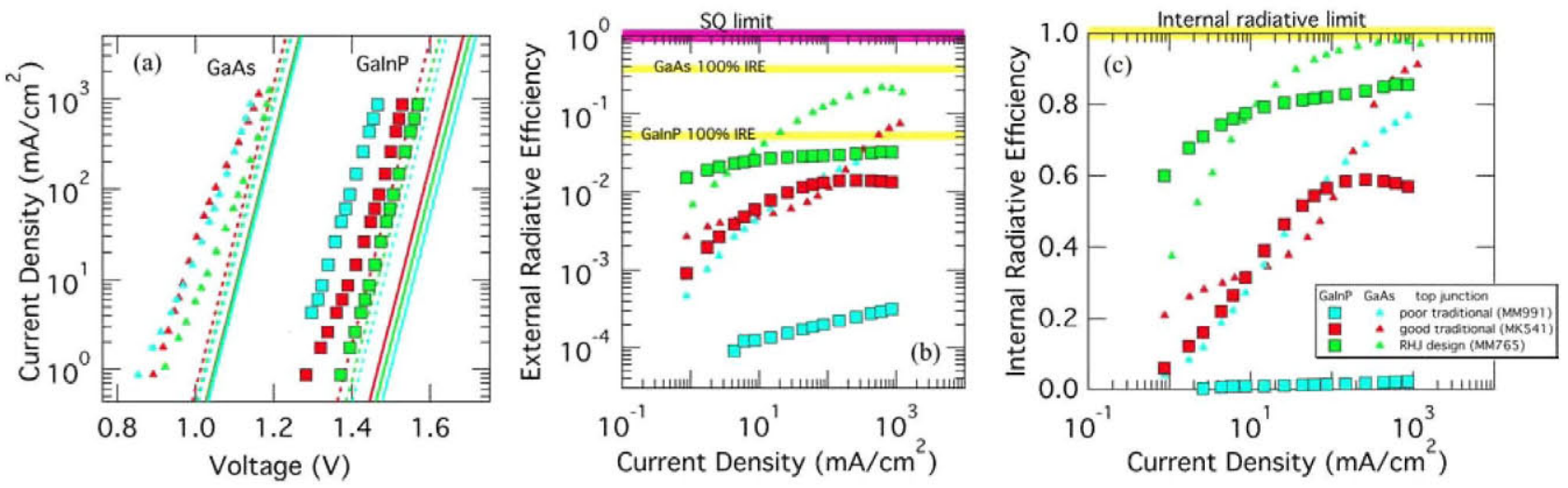

Fig. 5. EL measurements (markers) represented in terms of (a) voltage, (b) ERE, and (c) IRE of inverted 2-J GaInP/GaAs solar cells with SQ (solid lines or pink highlights) and internal (dashed lines or yellow highlights) radiative limits.

TABLE I

MODELED OPTICAL PARAMETERS USED IN (3) FOR TYPICAL DEVICE STRUCTURES WITH ARC

\begin{tabular}{|c|c|c|c|c|c|c|c|c|}
\hline \multirow{2}{*}{$\begin{array}{l}\text { Junction } \\
\text { Structure }\end{array}$} & \multicolumn{2}{|c|}{$\begin{array}{l}1.8-\mathrm{eV} \\
\mathrm{GaInP}\end{array}$} & \multicolumn{2}{|c|}{$\begin{array}{l}1.4-\mathrm{eV} \\
\mathrm{GaAs}\end{array}$} & \multicolumn{2}{|c|}{$\begin{array}{l}1.0-\mathrm{eV} \\
\text { InGaAs }\end{array}$} & \multicolumn{2}{|c|}{$\begin{array}{l}0.7-\mathrm{eV} \\
\text { InGaAs }\end{array}$} \\
\hline & $\bar{P}_{a b s}$ & $\bar{P}_{e s c}$ & $\bar{P}_{a b s}$ & $\bar{P}_{e s c}$ & $\bar{P}_{a b s}$ & $\bar{P}_{e s c}$ & $\bar{P}_{a b s}$ & $\bar{P}_{e s}$ \\
\hline Inverted $1 \mathrm{~J}$ & $91 \%$ & $2.3 \%$ & & & & & & \\
\hline Upright 1J & $64 \%$ & $1.5 \%$ & & & & & & \\
\hline Inverted $2 \mathrm{~J}$ & $64 \%$ & $1.5 \%$ & $96 \%$ & $1.0 \%$ & & & & \\
\hline Inverted $4 \mathrm{~J}$ & $66 \%$ & $1.4 \%$ & $89 \%$ & $0.7 \%$ & $81 \%$ & $1.1 \%$ & $96 \%$ & $1.1 \%$ \\
\hline
\end{tabular}

is somewhere in between these two extremes achieving almost $60 \%$ IRE at high current densities but falling rapidly below $85 \mathrm{~mA} / \mathrm{cm}^{2}$. These differences span about 2.5 orders of magnitude as quantified by the ERE in Fig. 5(b). In analyzing the dark JV in Fig. 5(a), it is clear that the RHJ top junction (green) is strongly $n=1$ dominated over the entire measurement range and quite close to the internal radiative limit. While the slope of the poor traditional top junction (blue) is nearly $n=1$, the performance is far from the internal radiative limit. The good traditional top junction (red) does approach its internal radiative limit and $\mathrm{n}=1$ behavior at high currents but transitions to nonradiatively dominated $n=2$ behavior at lower currents in agreement with the observations of nonlinear LC behavior.

The voltage difference between the RHJ (green) and good traditional (red) top junctions in Fig. 5(a) is dramatic at low currents but diminishes at high currents: $\Delta V=75 \mathrm{mV} @ 1.3 \mathrm{~mA} / \mathrm{cm}^{2}$; $\Delta V=49 \mathrm{mV} @ 15 \mathrm{~mA} / \mathrm{cm}^{2} ; \Delta V=40 \mathrm{mV} @ 865 \mathrm{~mA} / \mathrm{cm}^{2}$. Thus, the difference in the one-sun $V_{o c}$ is significant, but the voltage difference at the maximum power point, where the recombination current density is typically at least an order of magnitude lower than the photocurrent, is even greater. This indicates a tremendous advantage of the RHJ top junction compared with the traditional design at one-sun operation, but also a nontrivial advantage at concentrations as high as 1000 suns. It should be noted that small differences in the band gap of the top junctions through $\mathrm{CuPt}$ ordering of $\mathrm{GaInP}$ give rise to differences in predicted SQ and internal radiative limits that can account for some of this difference between devices (about $20 \mathrm{mV})$.

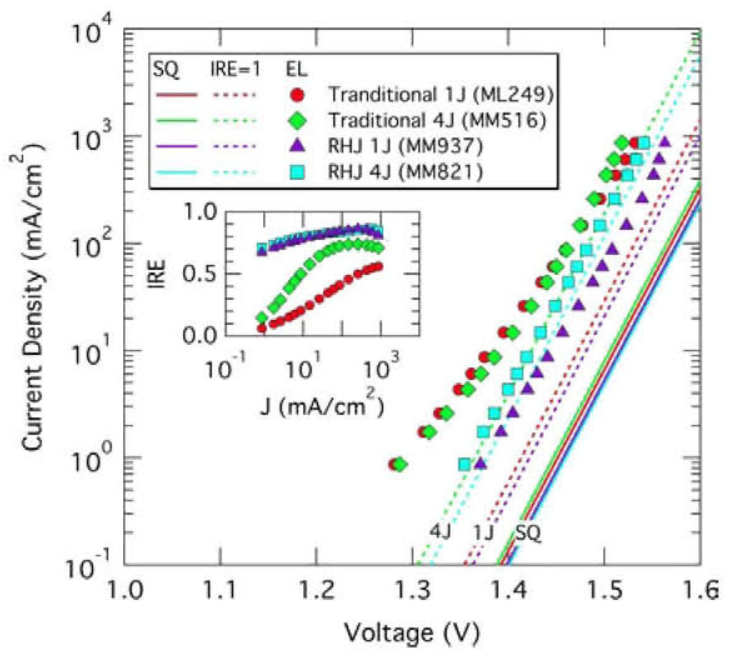

Fig. 6. GaInP junction dark JV (and IRE inset) from EL measurements (markers) within single and $4 \mathrm{~J}$ devices, comparing the traditional and RHJ designs. The SQ (solid) and internal (dashed) radiative limits are shown as lines.

While this study is focused on the GaInP top junction, it should be noted in passing that the GaAs junction performance (which is also a RHJ design in MM765) exceeds 97\% IRE at high currents, though begins to drop due to $n=2$ nonradiative recombination at slightly higher currents than the GaInP top junction. In addition, the GaAs junction of these inverted tandems clearly takes advantage of the gold BSR as evidenced by the fact that the internal radiative limit of the GaAs junction is much closer to the SQ limit than is the GaInP junction [see Fig. 5(a) and (b)]. Indeed, inverted 2J GaInP/GaAs tandem cells of this design currently holds the world record efficiencies for $2 \mathrm{~J}$ devices with $31.1 \%$ at one-sun AM1.5 global [16] and $34.1 \%$ at 467 -suns AM1.5 direct.

\section{Four Junction-Inverted Metamorphic Solar Cells}

We have also incorporated the RHJ design in both the top GaInP and second GaAs junctions of three- and four-junction (4J) IMM solar cells [21]. In 4J IMM solar cells, the top two junctions (1.8 eV GaInP and $1.4 \mathrm{eV} \mathrm{GaAs})$ are lattice-matched 

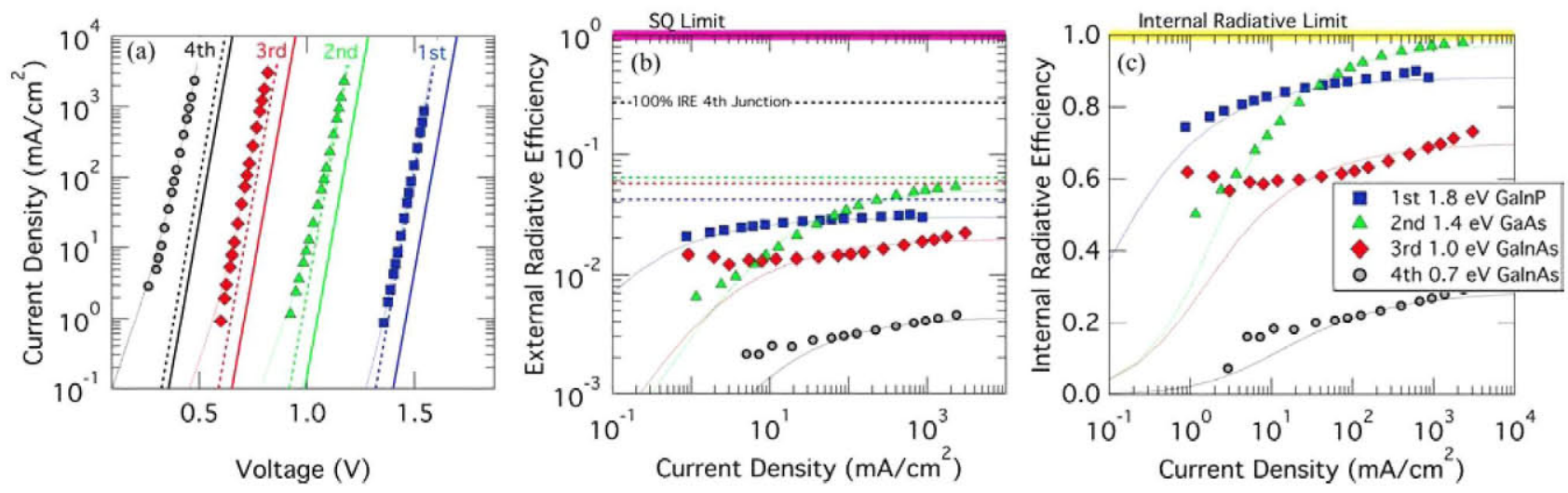

Fig. 7. EL measurements (markers) represented in terms of (a) voltage, (b) ERE, and (c) IRE of inverted 4J IMM solar cell (MM821) with SQ (solid lines or pink highlights) and internal (dashed lines or yellow highlights) radiative limits. The light dotted lines are an attempt to fit each junction to the two-diode model as used in fig. 8 .

to the GaAs substrate, while the third and fourth junctions are lattice-mismatched 1.0-eV In ${ }_{25} \mathrm{Ga}_{.75}$ As and $0.7-\mathrm{eV} \mathrm{In}_{.5} \mathrm{Ga}_{.5} \mathrm{As}$ junctions grown by metamorphic growth using two $\mathrm{Ga}_{x} \mathrm{In}_{1-x} \mathrm{P}$ compositionally graded buffer layers [22]. The coupled EL and LC techniques described in the previous section can be used in the same way for $3 \mathrm{~J}$ and $4 \mathrm{~J}$ IMM solar cells [20], but we focus on the analysis of a $4 \mathrm{~J}$ here.

Fig. 6 shows a comparison of the dark JV curves of four different GaInP junctions determined from EL measurements. The comparison shows both traditional and RHJ top junctions within $1 \mathrm{~J}$ and $4 \mathrm{~J}$ devices along with the SQ and internal radiative limits. Clearly both traditional junctions suffer from high $J_{02}$ from nonradiative recombination at low currents, while the RHJs do not (down to $\sim 1 \mathrm{~mA} / \mathrm{cm}^{2}$ ). In addition, the $J_{01}$ of the RHJ within the $4 \mathrm{~J}$ appears lower than $J_{01}$ in the traditional junction within the $4 \mathrm{~J}$ which results in an advantage even at high concentrations. While the IRE of the two RHJs are nearly identical (see Fig. 6), the optical environment of the inverted $1 \mathrm{~J}$ device including a gold BSR allows the internal radiative limit and actual results to more closely approach the SQ limit, compared with the RHJ in the $4 \mathrm{~J} \mathrm{IMM}$ where there is no reflector. However, the enhanced optical environment of the $1 \mathrm{~J}$ also magnifies the effects of any recombination losses, so while the data for the top RHJ in the 4J IMM are quite close to the internal radiative limit, the data for the $1 \mathrm{~J}$ RHJ are further away and the cell is not able to take full advantage of the gold BSR. The BSR of the $1 \mathrm{~J}$ traditional junction does not boost the voltage compared to the nonreflective optical environment of the traditional junction within the 4J IMM because the IRE is too low (except possibly at high currents).

Fig. 7 shows the equivalent 1$) \mathrm{V}(\mathrm{J}), 2) \eta_{\text {ext }}(J)$, and 3$) \eta_{\text {int }}(J)$ characteristics from (LC corrected) EL of all four junctions within a $4 \mathrm{~J}$ IMM using the RHJ design in the top two junctions. The IRE of the top two junctions in Fig. 7(c) are quite similar to the IRE of MM765 in Fig. 5(c), though the ERE of the second junction is reduced due to the change in optical environment from a gold BSR to subsequent junctions. In this $4 \mathrm{~J} \mathrm{IMM}$, only the fourth junction includes a gold BSR, which brings the internal radiative limit closer to the SQ limit, but the relatively low
IRE of the fourth junction does not result in much advantage of the BSR. Actually, the IRE of this fourth junction is surprisingly high considering the mid- $10^{6} \mathrm{~cm}^{2}$ threading dislocations (TDD) [22]. The IRE of the third junction is higher due to even lower TDD [23] $\left(\sim 10^{6} \mathrm{~cm}^{2}\right)$. In fact, at high concentrations (or current densities), the performance of the top three junctions in this $4 \mathrm{~J} \mathrm{IMM}$ are very close to the internal radiative limit. This means that the only way to dramatically improve the voltage of this device at high concentrations is to either improve the fourth junction by reducing the TDD or changing the optical environment of the top three junctions. We have considered techniques to incorporate omnidirectional selective reflectors by inserting distributed Bragg reflectors [24] or low index layers [25] between junctions to attain this improvement. However, the application of these interjunction reflectors has so far remained elusive. Instead, we note that the LC that naturally occurs without selective reflectors is also advantageous to the efficiency of $4 \mathrm{~J} \mathrm{IMM} \mathrm{solar} \mathrm{cells.} \mathrm{Characterization} \mathrm{of} \mathrm{the} \mathrm{LC} \mathrm{in} \mathrm{this} 4 \mathrm{~J}$ IMM shows relatively strong coupling between each adjacent junction, which counter-intuitively, also increases the voltage of the entire device [19], [26]. In addition, the effects of LC are advantageous to reduce spectral sensitivity under real world conditions [27], [28].

In Fig. 8 we show the performance of this $4 \mathrm{~J}$ IMM under concentration. Spectrally-adjustable [29] flash measurements (T-HIPSS) are in good agreement with the spectrally-adjustable one-sun measurements (OSMSS) and with the $\mathrm{V}_{\text {oc }}$ predicted from the sum of the EL measurements. This 4J IMM achieved an AM1.5 direct efficiency of $43.8 \%$ at 327 suns. Along with the measured data, the SQ and internal radiative limits are shown following the calculation procedure of Friedman et al. [19] to combine the performance of these ideal junctions. Since the SQ limit assumes that all the emitted light escapes the device, there cannot be any light available for $\mathrm{LC}$. The internal radiative limit, on the other hand, must result in LC that is determined by the optical geometry. The nonphysical calculated lines showing the internal radiative limit without LC are presented to gain insight into how much of the improvement is predicted to come from LC when ideal junctions exist in a real optical environment. Finally, 


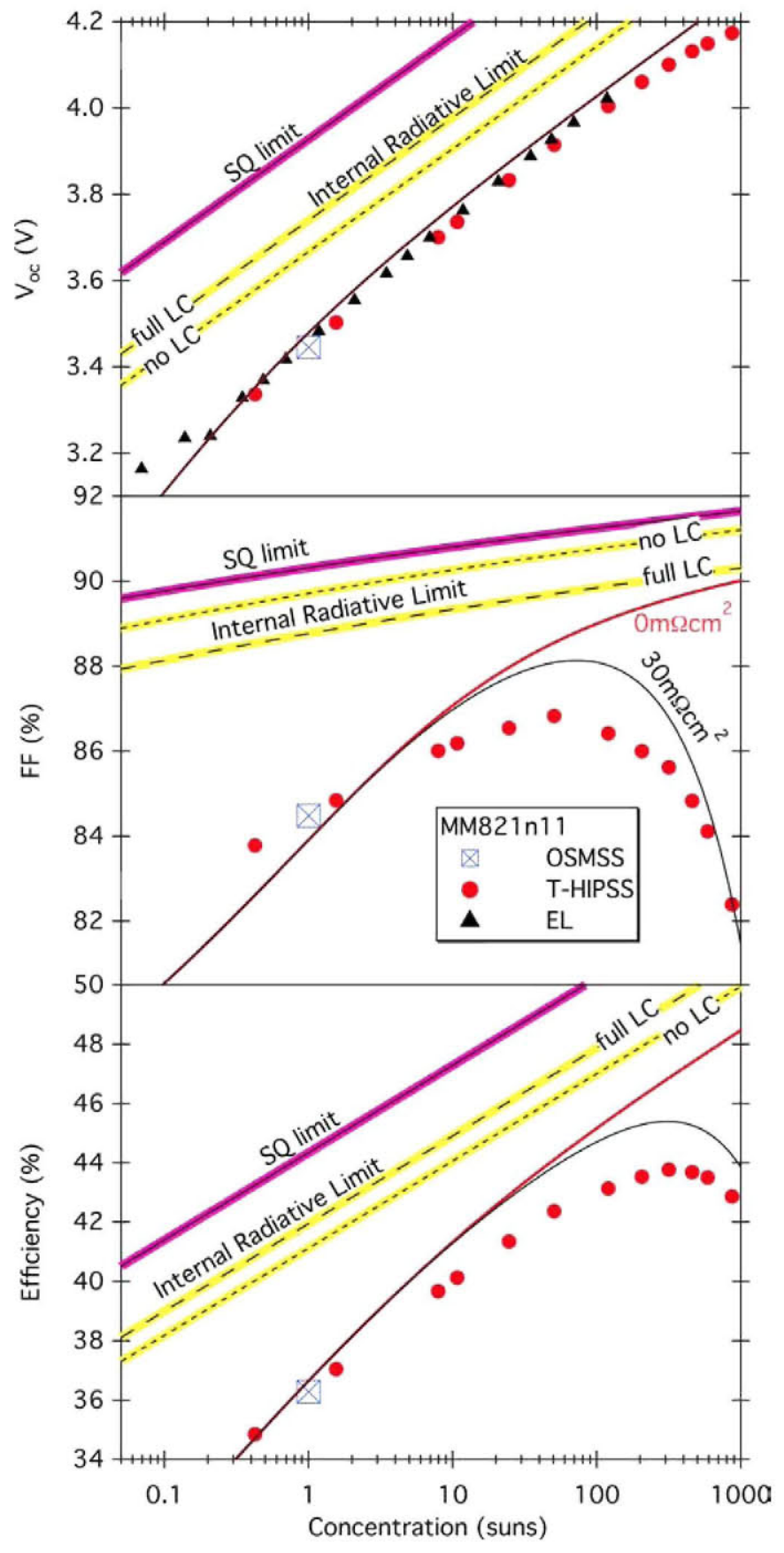

Fig. 8. 4J IMM solar cell performance (markers) as a function of concentration. SQ (pink) and internal (yellow) radiative limits are shown. The internal radiative limit is shown including and excluding the effects of LC. The black (finite series resistance) and red (no series resistance) lines show predictions based on a two-diode fit to the coupled EL and LC measurements.

the predictions of the coupled LC and EL analysis of the actual device, fitting the performance of each junction to a two diode $(n=1$ and $n=2)$ model (see dotted lines in Fig. 7) and again combining as described in [19], are presented as red and black lines in Fig. 8. While this prediction shows fairly good agreement with the flash data, it is not completely accurate because the nonradiative recombination cannot always be described by a simple $\mathrm{n}=2$ model. In particular, the third and fourth junctions in Fig. 7(c) are not as well-fit using this two-diode model as the top two junctions are.

\section{CONCLUSION}

Inverted multijunction solar cells using a RHJ design for the top GaInP junction dramatically reduce the nonradiative recombination of that junction resulting in high IRE. This high IRE manifests as higher overall device voltages at all concentrations, but especially at low concentration, as well as increased LC that also improves efficiency and decreases spectral sensitivity in real-world applications. We have demonstrated the usefulness of reciprocity relations and optical modeling to directly compare the measured performance of individual junctions within multijunction devices. We also compare the measured performance with the well-known SQ (or external radiative limit) and with an internal radiative limit that we propose to account for the optical environment.

\section{ACKNOWLEDGMENT}

The authors would like to thank W. Olavarria, M. Young, $\mathrm{S}$. Ward, and A. Duda for growth and fabrication of devices, T. Moriarty and C. Osterwald for official device results, and E. Yablonovitch for useful discussions.

\section{REFERENCES}

[1] W. Shockley and H. J. Queisser, "Detailed balance limit of efficiency of p-n junction solar cells," J. Appl. Phys., vol. 32, pp. 510-519, 1961.

[2] U. Rau, "Reciprocity relation between photovoltaic quantum efficiency and electroluminescent emission of solar cells," Phys. Rev. B, vol. 76 , pp. 085303-1-085303-8, 2007.

[3] O. D. Miller, E. Yablonovitch, and S. R. Kurtz, "Strong internal and external luminescence as solar cells approach the Shockley-Queisser limit," IEEE J. Photovoltaics, vol. 2, no. 3, pp. 303-311, Jun. 2012.

[4] M. A. Green, "Radiative efficiency of state-of-the-art photovoltaic cells," Prog. Photovoltaics, vol. 20, pp. 472-476, 2012.

[5] S. Roensch, R. Hoheisel, F. Dimroth, and A. W. Bett, "Subcell I-V characteristic analysis of $\mathrm{GaInP} / \mathrm{GaInAs} / \mathrm{Ge}$ solar cells using electroluminescence measurements," Appl. Phys. Lett., vol. 98, pp. 251113-1-251113-3, 2011.

[6] T. Kirchartz, U. Rau, M. Hermle, A. W. Bett, A. Helbig, and J. H. Werner, "Internal voltages in $\mathrm{GaInP} / \mathrm{GaInAs} / \mathrm{Ge}$ multijunction solar cells determined by electroluminescence measurements," Appl. Phys. Lett., vol. 92 , pp. 123502-1-123502-3, 2008.

[7] J. F. Geisz, M. A. Steiner, I. Garcia, S. R. Kurtz, and D. J. Friedman, "Enhanced external radiative efficiency for $20.8 \%$ efficient single-junction GaInP solar cells," App1. Phys. Lett., vol. 103, pp. 041118-1-041118-5, 2013.

[8] M. A. Steiner and J. F. Geisz, "Non-linear luminescent coupling in series-connected multijunction solar cells," Appl. Phys. Lett., vol. 100, pp. 251106-1-251106-5, 2012.

[9] M. A. Steiner, J. F. Geisz, I. Garcia, D. J. Friedman, A. Duda, and S. R. Kurtz, "Optical enhancement of the open-circuit voltage in high quality GaAs solar cells," J. Appl. Phys., vol. 113, pp. 123109-1-123109. $11,2013$.

[10] T. Takamoto, E. Ikeda, H. Kurita, and M. Ohmori, "Structural optimization for single junction InGaP solar cells," Sol. Energy Mater. Sol. Cells, vol. 35, pp. 25-31, 1994

[11] J. F. Geisz, "Application of III-V semiconductor heterostructures to optica chemical sensing," Ph.D. dissertation, Dept. Chem. Eng., Univ. WisconsinMadison, Madison, WI, USA, 1995.

[12] C. T. Sah, R. N. Noyce, and W. Shockley, "Carrier generation and recombination in p-n junctions and p-n junction characteristics," Proc. IRE, vol. 45 , pp. $1228-1243,1957$. 
[13] F. W. Ragay, E. W. M. Ruigrok, and J. H. Wolter, "GaAs-AlGaAs heterojunction solar cells with increased open-circuit voltage," in Proc. 1st World Conf. Photovoltaic Energy Convers., 1994, pp. 1934-1937.

[14] J. F. Geisz, S. R. Kurtz, M. W. Wanlass, J. S. Ward, A. Duda, D. J. Friedman, J. M. Olson, W. E. McMahon, T. Moriarty, and J. Kiehl, "High-efficiency GaInP/GaAs/InGaAs triple-junction solar cells grown inverted with a metamorphic bottom junction," Appl. Phys. Lett., vol. 91, pp. 023502-1-023502-3, 2007.

[15] E. Centurioni, "Generalized matrix method for calculation of internal light energy flux in mixed coherent and incoherent multilayers," Appl. Opt., vol. 44, pp. 7532-7539, 2005.

[16] M. A. Steiner, J. F. Geisz, I. Garcia, D. J. Friedman, A. Duda W. J. Olavarria, M. Young, D. Kuciauskas, and S. R. Kurtz, "Effects of internal luminescence and internal optics on $\mathrm{V}_{o c}$ and $\mathrm{J}_{\mathrm{sc}}$ of III-V solar cells," IEEE J. Photovoltaics, vol. 3, no. 4, pp. 1437-1442, Oct. 2013.

[17] M. P. Lumb, M. A. Steiner, J. F. Geisz, and R. J. Walters, "Analytical modeling of III-V solar cells close to the fundamental limit," Proc. SPIE, vol. 8981, pp. 898114-1-898114-9, 2014.

[18] M. A. Steiner, J. F. Geisz, T. E. Moriarty, R. M. France, W. E. McMahon, J. M. Olson, S. R. Kurtz, and D. J. Friedman, "Measuring IV curves and subcell photocurrents in the presence of luminescent coupling," IEEE J Photovoltaics, vol. 3, no. 2, pp. 879-887, Apr. 2013.

[19] D. J. Friedman, J. F. Geisz, and M. A. Steiner, "Analysis of multijunction solar cells current-voltage characteristics in the presence of luminescent coupling," IEEE J. Photovoltaics, vol. 3, no. 4, pp. 1429-1436, Oct. 2013

[20] J. F. Geisz, R. M. France, I. Garcìa, M. A. Steiner, and D. J. Friedman, "Device characterization for design optimization of 4 junction inverted metamorphic concentrator solar cells," AIP Conf. Proc., vol. 1616, pp. $114-118,2014$.

[21] R. M. France, J. F. Geisz, M. A. Steiner, I. García, W. E. McMahon, and D. J. Friedman, "Quadruple junction inverted metamorphic concentrator devices," IEEE J. Photovoltaics, 2014, to be published.
[22] R. M. France, I. García, W. E. McMahon, A. G. Norman, J. Simon, J. F. Geisz, D. J. Friedman, and M. J. Romero, "Lattice-mismatched $0.7-\mathrm{eV}$ GaInAs solar cells grown on GaAs using GaInP compositionally graded buffers," IEEE J. Photovoltaics, vol. 4, no. 1, pp. 190-195, Jan. 2014.

[23] R. M. France, J. F. Geisz, M. A. Steiner, B. To, M. J. Romero, W. J. Olavarria, and R. R. King, "Reduction of crosshatch roughness and threading dislocation density in metamorphic GaInP buffers and GaInAs solar cells," J. Appl. Phys., vol. 111, pp. 103528-1-103528-7, 2012.

[24] I. Garcia, J. Geisz, M. Steiner, J. Olson, D. Friedman, and S. Kurtz, "Design of semiconductor-based back reflectors for high $\mathrm{V}_{\mathrm{oc}}$ monolithic multijunction solar cells," in Proc. 38th IEEE Photovoltaic Spec. Conf., 2012, pp. 002042-002047.

[25] I. García, C. F. Kearns-McCoy, J. S. Ward, M. A. Steiner, J. F. Geisz, and S. R. Kurtz, "Back reflectors based on buried $\mathrm{Al}_{2} \mathrm{O}_{3}$ for enhancement of photon recycling in monolithic, on-substrate III-V solar cells," Appl. Phys. Lett., vol. 105, pp. 133507-1-133507-5, 2014.

[26] D. J. Friedman, J. F. Geisz, and M. A. Steiner, "Effect of luminescent coupling on the optimal design of multijunction solar cells," IEEE J. Photovoltaics, vol. 4, no. 3, pp. 986-990, May 2014

[27] A. S. Brown and M. A. Green, "Radiative coupling as a means to reduce spectral mismatch in monolithic tandem solar cell stacks-theoretical considerations," in Proc. 29th IEEE Photovoltaic Spec. Conf., 2002, pp. $868-871$

[28] I. García, W. E. MeMahon, M. A. Steiner, J. F. Geisz, A. Habte, and D. J. Friedman, "Optimization of multijunction solar cells through indoor energy yield measurements," IEEE J. Photovoltaics, 2014, to be published.

[29] C. R. Osterwald, M. W. Wanlass, T. Moriarty, M. A. Steiner, and K. A. Emery, "Empirical procedure to correct concentrator cell efficiency measurement errors caused by unfiltered Xenon flash solar simulators," in Proc. 40th IEEE Photovoltaic Spec. Conf., 2014.

Authors' photographs and biographies not available at the time of publication. 